\title{
Noninvasive blood glucose monitoring system based on near-infrared method
}

\author{
Mustafa Ayesh Al-dhaheri, Nasr-Eddine Mekkakia-Maaza, \\ Hassan Mouhadjer, Abdelghani Lakhdari \\ LMSE, Laboratoire Microsystèmes et Systèmes Embarqués Département d’Electronique, Faculté de Génie Electrique, \\ USTO MB, BP1505 El Mnaouer, 31000 Oran Algérie.
}

\section{Article Info \\ Article history: \\ Received Jun 27, 2019 \\ Revised Oct 27, 2019 \\ Accepted Nov 5, 2019}

\section{Keywords:}

Blood glucose

Near-infrared spectroscopy

Noninvasive method

\begin{abstract}
Diabetes is considered one of the life-threatening diseases in the world which need continuous monitoring to avoid the complication of diabetes. There is a need to develop a non-invasive monitoring system that avoids the risk of infection problems and pain caused by invasive monitoring techniques. This paper presents a method for developing a noninvasive technique to predict the blood glucose concentration (BCG) based on the Near-infrared (NIR) light sensor. A prototype is developed using a finger sensor based on LED of $940 \mathrm{~nm}$ wavelength to collect photoplethysmography (PPG) signal which is variable depending on the glucose concentration variance, a module circuit to preprocess PPG signals is realized, which includes an amplifier and analog filter circuits, an Arduino UNO is used to analog-to-digital conversion. A digital Butterworth filterer is used to remove PPG signal trends, then detect the PPG data peaks to determine the relationship between the PPG signal and (BCG) and use it as input parameters to build the calibration model based on linear regression. Experiments show that the Root Mean Squares Error (RMSE) of the prediction is between $8.264 \mathrm{mg} / \mathrm{dL}$ and $13.166 \mathrm{mg} / \mathrm{dL}$, the average of RMSE is about $10.44 \mathrm{mg} / \mathrm{dL}$ with a correlation coefficient $\left(\mathrm{R}^{\wedge} 2\right)$ of 0.839 , it is observed that the prediction of glucose concentration is in the clinically acceptable region of the standard Clark Error Grid (CEG).
\end{abstract}

Copyright $\left({ }_{0} 2020\right.$ Institute of Advanced Engineering and Science. All rights reserved.

\section{Corresponding Author:}

Mustafa Ayesh Al-dhaheri,

Laboratoire des Microsystèmes et Systèmes Embarqués L.M.S.E,

Université des Sciences et de la Technologie d'Oran, Mohamed Boudiaf, USTO-MB,

BP 1505 El M'naouer, 31000 Oran, Algérie.

Email: mu.eldhaheri@gmail.com or mustafaayeshahmed.eldhaheri@univ-usto.dz

\section{INTRODUCTION}

Diabetes has become a common disease in modern society. It is a chronic disease in which blood glucose concentration fluctuates from its normal range $(90-140 \mathrm{mg} / \mathrm{dl})$ causing a significant impact on health [1-2]. According to a study made by the International Diabetes Federation (IDF) in 2014, 387 million people had diabetes; by 2035 this will rise to 600 million [3]. Long-term diabetes is dangerous as it can lead to serious health problems such as cardiovascular diseases, damage of blood vessels, stroke, blindness, chronic kidney failure, nervous system diseases, amputation of a foot due to ulceration and early death [4]. Diabetes complications can be prevented by regular blood glucose monitoring and adhering to the doctor's guidance regarding diet, medication, and exercise. Currently, most of commercially available glucose measurement devices are invasive that require finger pricking to extracting the blood from forearm and doing chemical analysis using a test strip which is inserted into an electronic glucometer to determine the level of blood glucose, these procedures for this invasive method are considered as disadvantages because it is painful, uncomfortable, and expensive in the long term. 
Latterly, a biosensor has been developed to determine the glucose concentration in a liquid specimen [5-6]. This biosensor is low-cost, portable, and friendly to the environment. The main disadvantage of this biosensor is that needs a liquid specimen and a lot of effort is required to extract the liquid from the body that rests inconvenient. Non-invasive methods are more desirable and excellent alternatives to these invasive methods because it is more convenient and comfortable for patients and less expensive. There are a number of techniques employed for blood glucose noninvasive measurement that has been proposed [7]. Optical methods are one of the most promising methods that can be used for non-invasive blood glucose measurement. This technique has been applied to various body parts: finger, palm, arm, forearm, earlobe, check, etc. The concept is to irradiate a particular wavelength range of optical radiation on the avascular region of the body and obtain the transmission Photoplethysmography signal (PPG). Near-infrared (NIR) spectroscopy which uses light in $(750-2500 \mathrm{~nm})$ wavelength range is one of the most widely recommended optical technique because of its high penetration in skins, very simple and affordable equipment as compared to other optical methods [7-9] different bands in these regions are combination overtone band $(2000-2500 \mathrm{~nm})$, first overtone band (1400-2050 nm) and second overtone absorption bands $(750-1550 \mathrm{~nm})$ [10]. The absorbance of the glucose bonds $(\mathrm{C}-\mathrm{H}, \mathrm{O}-\mathrm{H})$ is strong in combination and first overtone regions but there are some disadvantages in these regions due to three causes. Firstly, the drastic increase in the cost of components and secondly the strong absorption due to other components like water and lastly the scattering in fatty tissue. in the second overtone region which is used in the present work, the components are less affordable, tissue absorption is lower and light penetration is higher, but glucose has weaker absorption and it needs the electronic noise in the analog sensing circuit to be suppressed much below the signal from the sensor output $[11,12]$.

The key to use NIR spectroscopy for developing glucose measurement sensor is to model the relationship between the PPG signal and BGC. As the PPG is more susceptible for the motion artifact induced errors, it is necessary that this noise has to be filtered before giving it to the regression model for estimating glucose levels. In this work, the development of a non-invasive blood glucose measuring prototype is performed using the optical techniques based on NIR light source $(940 \mathrm{~nm})$ where glucose has an acceptable absorption as compared to other chromophores of blood. The output of the detector is preprocessing with analog circuit and Butterworth is used for smoothing the PPG signal. The method uses peaks of the PPG and a private individual calibration model for each subject is built between PPG and the blood glucose concentration used linear regression. The experiments in this work were carried out with 10 healthy volunteers without any history of hypertension in the age group of 20-36 years (including both male and female volunteers). The private individual calibration model has been built for each subject separately from each other to avoid the influence due to the difference in the properties between persons such as the circumference of a subject's finger, the different body fluid concentration, and the roughness of the skin that can cause the scattering of light, etc. These different properties could influence PPG reading. Moreover, the calibration models have been built in comfortable conditions of measurement during the PPG reading to improve accuracy and repeatability.

The subsequent sections are organized as follows: the principle of glucose measurement using the NIR method is given in Section 2. Material and methods including the selection of wavelengths, analog sensing circuit, experimental protocols, and data treatment are described in section 3. Results and discussion are presented in section 4 and Section 5 concludes the paper.

\section{PRINCIPLE OF BLOOD GLUCOSE MEASUREMENT}

The NIR region of the electromagnetic spectrum is normally understood to range (from $780 \mathrm{~nm}$ to $2500 \mathrm{~nm}$ ). As shown in Figure 1 the molecular formula for glucose molecule is C6H12O6, which consists of $\mathrm{C}-\mathrm{H}, \mathrm{O}-\mathrm{H}$ and $\mathrm{C}=\mathrm{O}$ bonds [11]. The presence of these bonds causes the absorption of NIR light in blood or other human blood components. The NIR transmittance spectroscopy can be used for earlobes and fingertips but for forearms and cheeks, reflective spectroscopy is used because of the very low penetration of NIR [13]. When a beam of near-infrared light is focused on the human fingertip and reaches the corresponding detector sensor, the absorption of skin tissue and blood will weaken the beam, thus the photoelectric signal of detector sensors will be attenuated.

The absorption is described by the Beer-Lambert law:

$$
I=I_{0} e^{-\mu_{e f f} L}
$$

Where $I_{0}$ : Incident light intensity .

I: Transmitted light intensity.

L: Light path Length through the solution. 
$\mu_{e f f}$ : The effective attenuation coefficient, defined in (2)

$$
\mu_{e f f}=\left[3 \mu_{a}\left(\mu_{a}+\mu_{s}^{\prime}\right)\right]^{1 / 2}
$$

Light transport in tissue is expressed in terms of the absorption coefficient $\mu_{a}$ and reduced scattering coefficient $\mu_{s}^{\prime}$. The $\mu \mathrm{a}$ is related to the tissue chromophores concentration and is given by (3).

$$
\mu_{a}=2.303 \varepsilon \mathrm{C} \mathrm{cm}^{-2}
$$

Where $\varepsilon$ : Extinction coefficient.

$\mathrm{C}$ : Liquid concentration that means blood glucose concentration in our work.

Value of $\mu_{a}$ changes with variation in glucose concentration. The reduced scattering coefficient $\left(\mu_{s}^{\prime}\right)$ is given by (4).

$$
\mu_{s}^{\prime}=\mu_{s}[1-\mathrm{g}]
$$

Where $\mathrm{g}$ is the anisotropy factor and $\mu_{s}$ is the scattering coefficient. As shown in Figure 2. The variation in blood glucose concentration causes a change in the intensity of light scattered from the tissue. The glucose concentration of extracellular fluid( ECF) increases when it increases in blood while that of tissue cells remains constant and the refractive index of ECF $(\eta E C F)$ approaches that of tissue cells (approximately 1.350-1.460) hence leading to the decrease in the properties of scattering $[10,14,15]$.

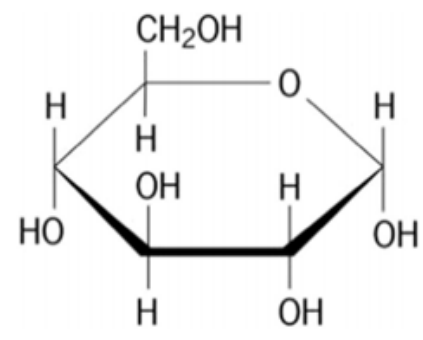

Figure 1. Molecular structure of glucose

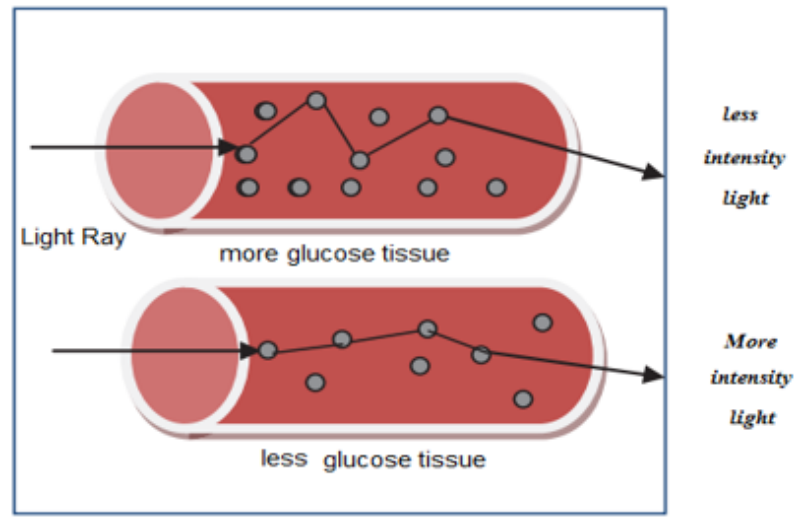

Figure 2. Schematic decription of effect of glucose on light path

\section{MATERIAL AND METHODS}

\subsection{The selection of wavelengths}

A study has been conducted to determine the glucose absorption spectrum in order to choose the corresponding wavelengths. In the wave ranges of [900 - 980] $\mathrm{nm}$ exists the near-infrared spectrums absorption of glucose as shown in Figure 3 the glucose molecule exhibits peak absorption at $939 \mathrm{~nm}$ which is very close to $940 \mathrm{~nm}[10]$. Moreover, this wavelength is selected in this rang to utilize the advantage of therapeutic "therapeutic window" where tissue absorption is low and light penetration is high. Although 
glucose has less absorption in this range, it requires a depth of penetration which can be realized in this wavelength due to the minimum attenuation of the optical signal by other constituents of the blood like water, platelets, red blood cells, etc., in addition, the electronic noise in the analog sensing circuit has been realized to be suppressed much below the signal from the sensor output $[10,11,16]$

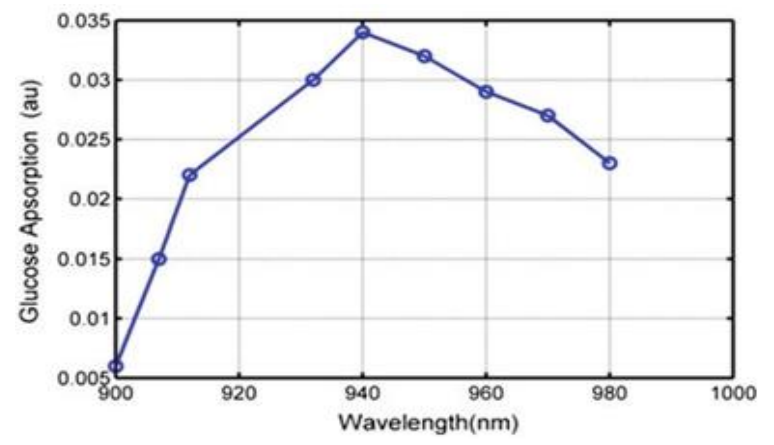

Figure 3. Glucose absorption spectra between 900 and $980 \mathrm{~nm}$

\subsection{Photoplethysmography (PPG)}

The variations in the volume of blood flow can be detected optically by the PPG sensors, which is a low cost and non-invasive technique that makes measurements at the skin surface. As shown in Figure 4 the PPG signal is composed of two parts: 1) Direct current (DC) component: it corresponds to the transmitted optical signal of the near-infrared light absorption of fingertip skin, muscle, bone, and venous blood. The DC component essentially in a short time remains constant. 2) Alternating current (AC) component: it corresponds to the transmitted optical signal of the near-infrared light absorption fingertip arterial blood. The AC component will change periodically with the heart rate [17, 18, 19]. Although the origins of the components of the PPG signal are not fully understood, it is generally accepted that they can provide valuable information about the cardiovascular system. It can monitor the heartbeat by means of differences in light absorption during systole and diastole of blood circulating. An experimental setup has been developed for non-invasive measurement of blood glucose levels based on PPG signal with some modifications during heart rate variability.

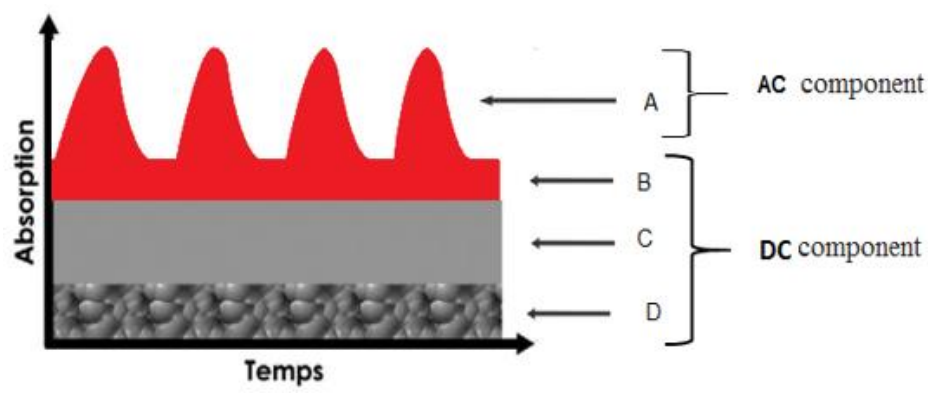

Figure 4. Principle of the PPG signal. A: Variable light absorption by reason of the change in arterial blood volume. B: constant due to the light absorption part of the non-pulsatile arterial blood. C: constant light absorption related to venous blood. D: constant light absorption associated with the tissue, bones.

\subsection{Analog sensing circuit}

The block diagram of the noninvasive blood glucose measurement system is shown in Figure 5. It consists of two parts: 1) The module to collect and preprocess the PPG signal. 2) The module to construct the calibration model, the Butterworth filter and linear regression are applied to develop the model, as discussed in the following section. In the module to collect and preprocess the PPG signal, it consists of an optical Sensor with LED as the light source and photodiode as the detector to detect the attenuation of NIR light, which passes through the fingertip with the change of blood glucose concentration. The detector converts this light into an equivalent current and a high pass filter of cutoff frequency $0.8 \mathrm{~Hz}$ is realized to

Noninvasive blood glucose monitoring system based on near-infrared method (Mustafa Ayesh Al-dhaheri) 
remove baseline drift or low-frequency signals. Twin-T notch filter with a potentiometer is realized to detect and remove $50 \mathrm{~Hz}$ power line interference. Then the LM324 amplifier is used for amplification of the signal. After necessary amplification, a low pass filter of cutoff frequency $8 \mathrm{~Hz}$ is realized to remove high-frequency components. The Analog circuit is represented in Figure 6. The analog signal is converted into digital with the help of Arduino-UNO connected to a PC to acquire the signal and send the signal to the PC to be processed by MATLAB [20, 21]. Figure 7 shows the PPG waveform.

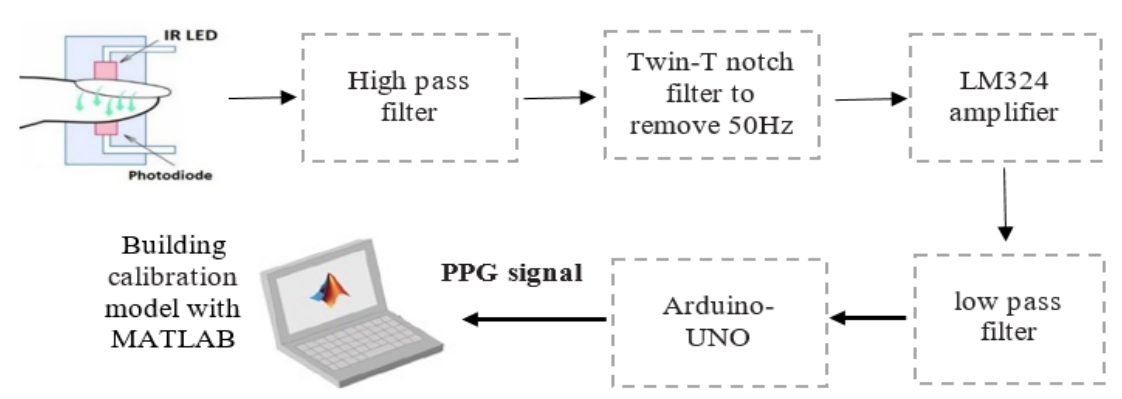

Figure 5. Block diagram of the proposed noninvasive blood glucose measurement system

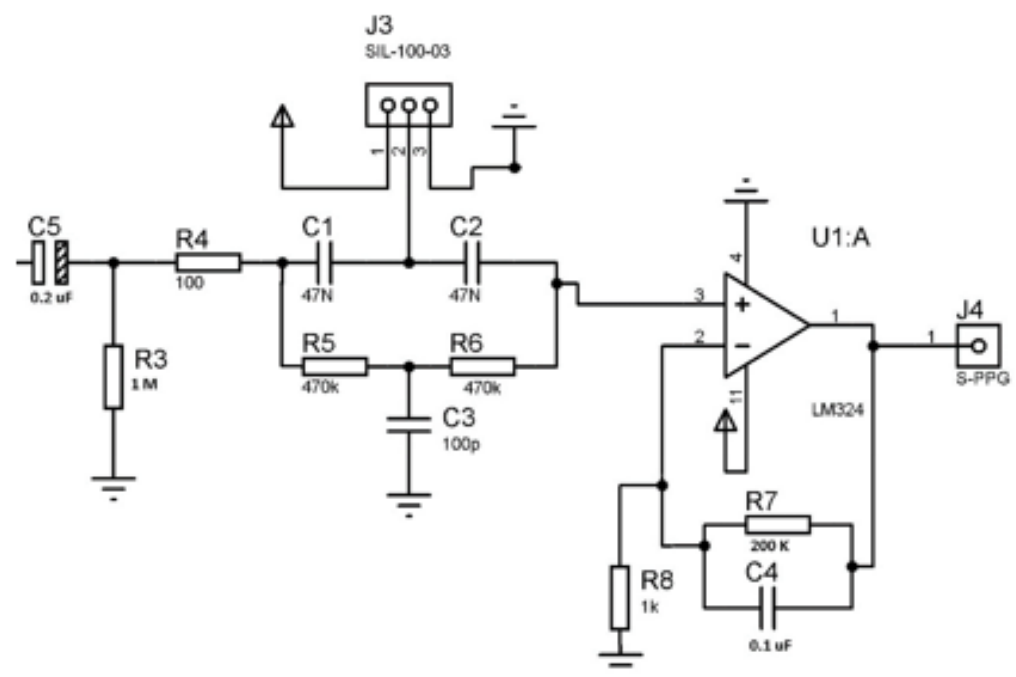

Figure 6. Analog circuit for preprocess of PPG signal

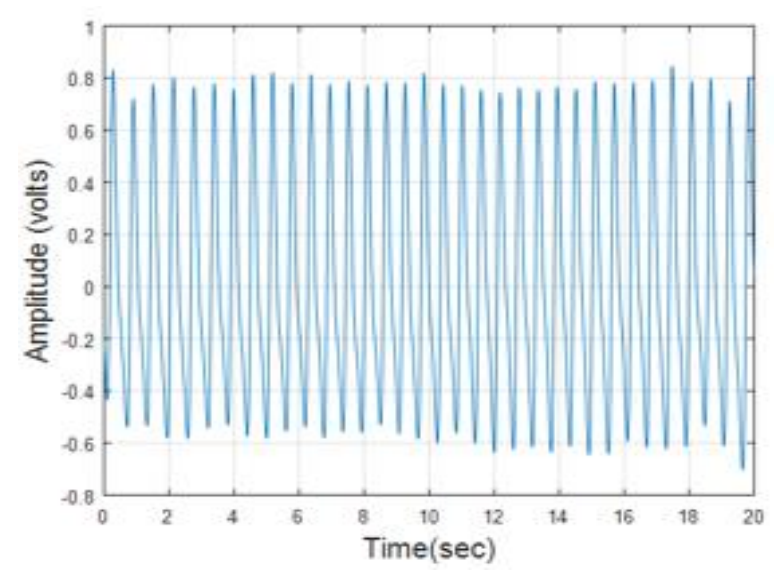

Figure 7. The PPG waveform 


\subsection{Experimental protocol}

In this work, a series of experiments were carried out to build the calibration model and prediction of glucose concentrations using a noninvasive glucose sensor. Ten healthy volunteers were recruited for these experiments in the age group of 20-36 year (including both male and female volunteers) without any history of hypertension. Blood glucose measurements were made at different times during several days before and after several meals which produce different amounts of glucose levels in the blood in order to test the ability of our prototype to determine the blood glucose levels during these different levels. The experiment was conducted using a commercial invasive glucometer"Glucoleader Check 3" with our prototype to build the calibration model and benchmark the performance of our developed system. During the PPG reading used our prototype, subjects are asked to sit comfortably on a chair and not allowed to move and the indoor temperature and humidity were maintained at comfortable levels for getting a stable and accurate PPG signal. The sensor is placed on the finger as shown in Figure 8 for collecting PPG single. Fifteen measurements have been made for each subject before and after several meals, 15 stable PPG signals were collected and the invasive blood glucose reading was performed immediately with each reading of the PPG signal for getting the reference values of blood glucose concentration, including ten sets of PPG signals and ten invasive values reading have been used to construct the private individual calibration model using the regression analysis model for each volunteer, while those in last PPG signals and invasive values reading have been treated as validation data set to test the performance of the measurement model.

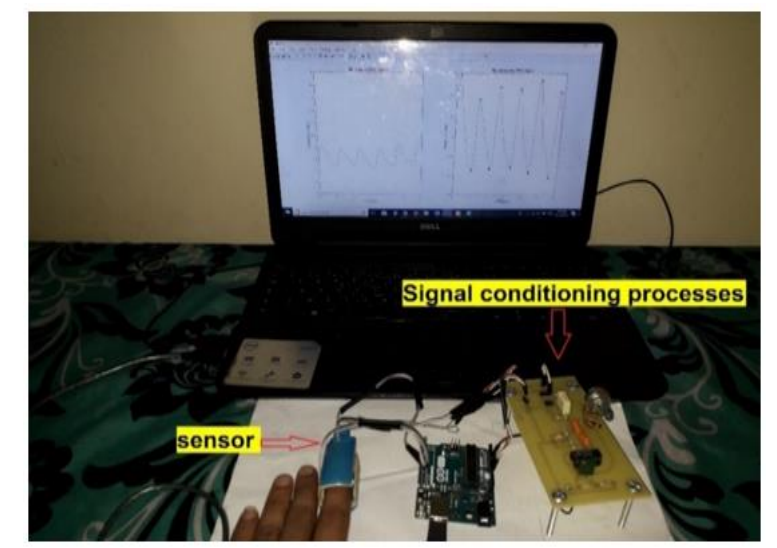

Figure 8. The experimental setup of the sensing prototype

\subsection{Data treatment}

There are some properties that vary from person to person which could influence the PPG reading such as the circumference of a subject's finger, the different body fluid concentration, and the roughness of the skin that can cause the scattering of light, etc. In order to avoid this influence and improve the system performance, each person is required to perform ten measurements in order to construct his private individual calibration model, the blood glucose concentration of each person is then predicted based on his private individual calibration model. After smoothing the PPG signal using the Butterworth filter [22, 23], the calibration model between the PPG data and reference values of BGC was built as shown in Figure 9.

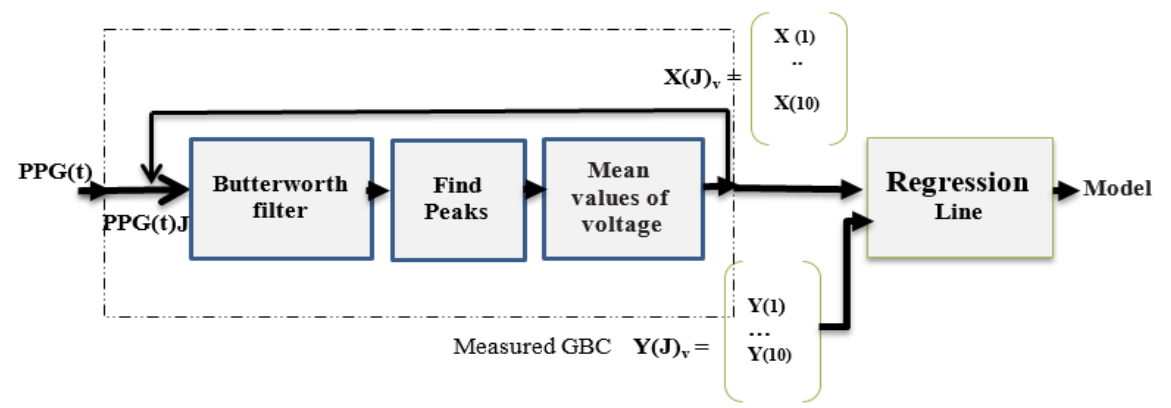

Figure 9. Construction procedure of calibration model using linear regression

Noninvasive blood glucose monitoring system based on near-infrared method (Mustafa Ayesh Al-dhaheri) 
The mean value of voltage is calculated from the peaks of PPG data obtained, as there exists a functional relationship between the PPG signal and blood glucose level [24], the voltage intensity of PPG signal changes with variation in glucose concentration. Ten means voltage is calculated from ten PPG readings for the same subject and put into a vector and put the ten reals GCB readings for the same subject into a vector, then used the two vectors as input data for constructing the regression model.

$$
\begin{aligned}
& \mathrm{Xv}=\left[\begin{array}{lll}
\mathrm{X}(1) \ldots \mathrm{X}(10)
\end{array}\right] \\
& \mathrm{Yv}=\left[\begin{array}{lll}
\mathrm{Y}(1) \ldots \ldots \mathrm{Y}(10)
\end{array}\right]
\end{aligned}
$$

Where Xv: Vector voltages PPG readings.

Yv: Vector reals GCB readings.

To construct the regression model, the regression line must be calculated. The general linear regression model is given by:

$$
\mathrm{Y}=\mathrm{mX}+\mathrm{b}
$$

Where Y: The predict blood glucose concentration.

$\mathrm{X}$ : The voltage of the PPG signal.

The (m) and (b) are the regression coefficients which is given by:

$$
\begin{aligned}
& m=\frac{\mathrm{X}_{\mathrm{v}}^{\prime} \mathrm{Y}^{\prime}{ }_{\mathrm{v}}-\left(\mathrm{X}_{\mathrm{v}} \mathrm{Y}_{\mathrm{v}}\right)^{\prime}}{\left(\mathrm{X}_{\mathrm{v}}\right)^{2}-\left(\mathrm{X}_{\mathrm{v}}{ }^{2}\right)^{\prime}} \\
& b=m \mathrm{X}^{\prime}{ }_{\mathrm{v}}+\mathrm{Y}_{{ }_{\mathrm{v}}}^{\prime}
\end{aligned}
$$

Where $\mathrm{X}_{\mathrm{v}}^{\prime}$ : the mean of vector voltages PPG readings.

$\mathrm{Y}^{\prime}{ }_{\mathrm{v}}$ : the mean of vector reals GCB readings.

\section{RESULTS AND DISCUSSION}

To obtain the calibration model between reals GCB readings and PPG voltages readings, regression analysis was done in MATLAB. Figure 10 shows a regression analysis example of a subject which indicates that voltage PPG reading increases with the increase of real BGC. The regression coefficients were calculated from (8) and (9) and were substituted in (7) for the prediction of non-invasive glucose concentration. When the system was fully calibrated, the validation data sets for each subject were used to test the performance of the measurement model. The measurements presented in Table 1, this table shows some of the benchmarking test results between commercially invasive glucometer and our proposed non-invasive NIR sensor. The comparison between the predicted values and the real values of blood glucose concentration is shown in Figure 11.

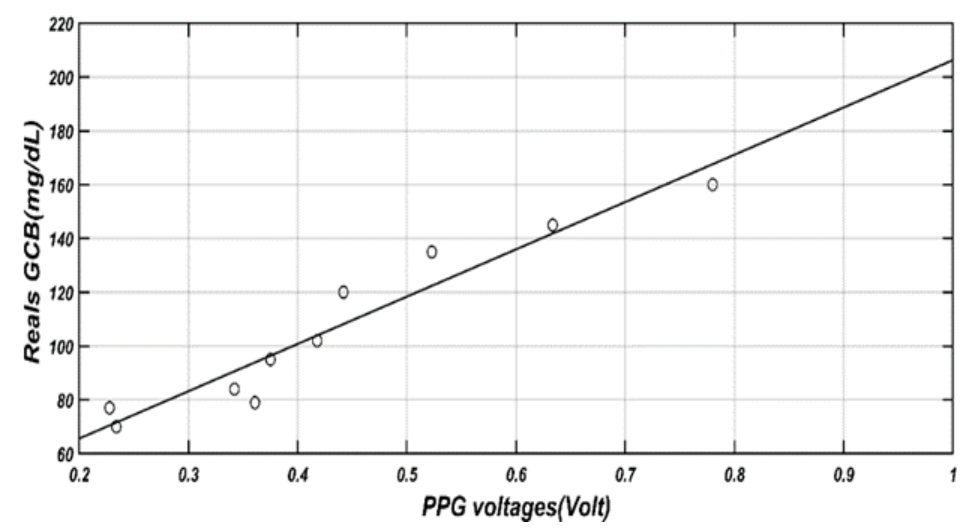

Figure 10. Regression analysis for one subject 


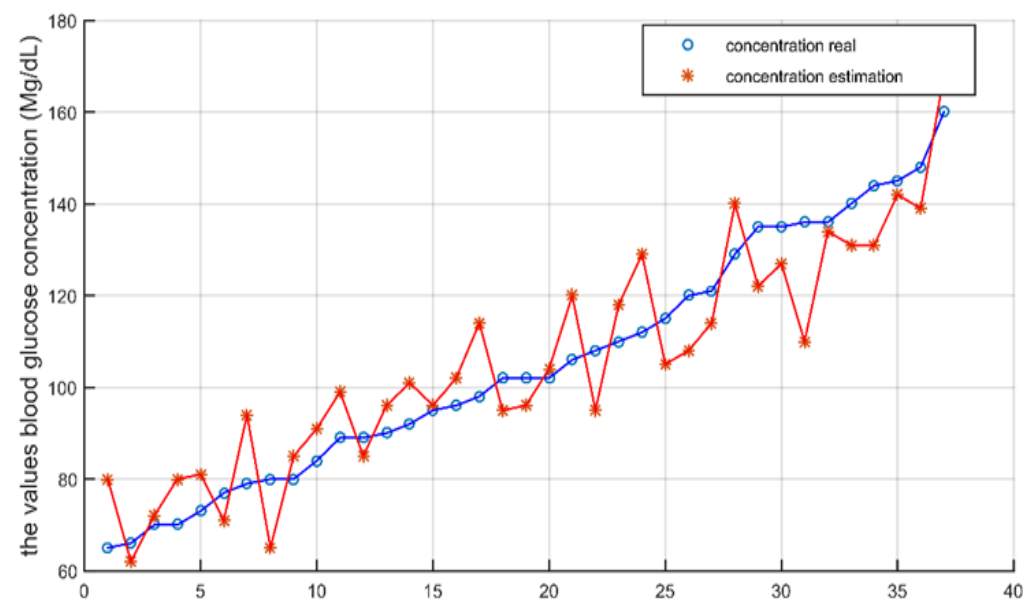

Figure 11. Comparison between the predicted values and the real values of BGC

The performance of our proposed non-invasive glucose sensor prototype is evaluated according to Root Mean Squares Error (RMSE) and correlation coefficients $\left(R^{2}\right)$ :

$$
\begin{aligned}
& R M S E=\sqrt{\frac{\sum_{i=1}^{n}\left(X_{\mathrm{i}}-Y_{\mathrm{i}}\right)^{2}}{n}} \\
& R^{2}=1-\frac{\sum_{i=1}^{n}\left(X_{\mathrm{i}}-Y_{\mathrm{i}}\right)^{2}}{\sum_{i=1}^{n}\left(X_{\mathrm{i}}-Y^{\prime}\right)^{2}}
\end{aligned}
$$

Where $X_{\mathrm{i}}$ : is the predicted blood glucose concentration.

$Y_{\mathrm{i}}$ : The real GCB reading .

$Y^{\prime}$ : the mean of real GCB reading.

Table 1. Comparison of results between commercially invasive glucometer and our proposed non-invasive NIR sensor

\begin{tabular}{ccccc}
\hline Subject & Non-invasive NIR sensor & Invasive glucometer $(\mathrm{Mg} / \mathrm{dl})$ & $\mathrm{RMSE}(\mathrm{Mg} / \mathrm{dl})$ & $R^{2}$ \\
\hline \multirow{4}{*}{$\mathrm{A}$} & 168 & 160 & & \\
& 104 & 102 & 8.264 & 0.923 \\
& 95 & 96 & & \\
$\mathrm{~B}$ & 72 & 70 & & \\
& 139 & 148 & 9.293 & 0.881 \\
& 134 & 136 & & \\
$\mathrm{C}$ & 96 & 90 & & \\
& 80 & 70 & 11.217 & 0.7352 \\
& 114 & 121 & & \\
$\mathrm{D}$ & 105 & 115 & & \\
& 92 & 101 & & \\
& 81 & 73 & & \\
\end{tabular}

RMSE and $\mathrm{R}^{\wedge} 2$ are calculated for each subject calibration model as shown in Table 1, the averages of root mean square error and correlation coefficients $\left(\mathrm{R}^{\wedge} 2\right)$ were as follows: $10.44 \mathrm{mg} / \mathrm{dL}, 0.8392$. Most researchers and clinicians tend to use Clarke error grid which is a frequently method used for evaluating the clinical authentication of glucose predictions[25], as shown in Figure 12 all noninvasive values are within the acceptable range. The results obtained from experimentations were encouraging and confirmed the correctness of the proposed method. Comparing our results with the previous articles; the results obtained from the proposed method revealed that the values of performance measures were

Noninvasive blood glucose monitoring system based on near-infrared method (Mustafa Ayesh Al-dhaheri) 
significantly ameliorated as compared to the previous works. Table 2 shows a comparison between the proposed system and previous articles. The obtained RMSE value is lower than previously and $\mathrm{R}^{\wedge} 2$ is higher which indicates an improvement in the accuracy of the results due to the amelioration achieved by building a private individual calibration model for each subject separately from each other, then the prediction of the blood glucose concentration for each subject is performed based on his private individual calibration model for avoiding the disadvantage of various properties from person to another which could influence on the PPG reading such as the circumference of a subject's finger, skin roughness which can cause light scattering and different body fluid concentration, etc., which is difficult to avoid with universal calibration model. The data collection was carried out in comfortable conditions for getting a stable and accurate PPG signal using our proposed system which is developed with very low-cost materials. In this preliminary phase study, 10 persons were selected as subjects. In the near future, we will plan an experimental procedure for more subjects. In addition, we will use different wavelengths and multiple regression analyses for further improvement of the proposed system.

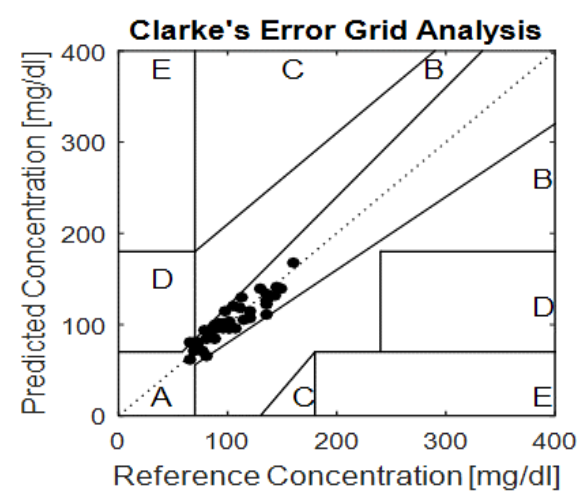

Figure 12. Clarke Grid analysis of results

Table 2. Comparisons between the proposed system and previous articles

\begin{tabular}{|c|c|c|c|c|}
\hline Reference & Method & Wavelength (nm) & Cost & Summary RMSE \& $R^{2}$ \\
\hline J. Yadav [10] & $\begin{array}{l}\text { Diffuse } \\
\text { reflectance }\end{array}$ & 940 & Low & $\begin{array}{l}\mathrm{RMSE}=14.9248 \mathrm{mg} / \mathrm{dl} \\
R^{2}=0.87\end{array}$ \\
\hline B. Javid [26] & Refraction \& transmission & $940,1550,1650$ & Low & $\mathrm{RMSE}=18.52 \mathrm{mg} / \mathrm{dL}$ \\
\hline H. Ali [28] & Refraction \& transmission & 650 & Low & Not reported \\
\hline S. Haxha [29] & Absorption & 940 & High & Not reported \\
\hline X. Li [30] & $\begin{array}{l}\text { Transmission } \\
\text { Diffuse }\end{array}$ & 1310 & Medium & Not reported \\
\hline E. Guevara [31] & $\begin{array}{l}\text { reflectance } \\
\text { NIRS and } \\
\text { impedance } \\
\text { spectroscopy }\end{array}$ & $700-1000 \mathrm{~nm}$ & High & $\mathrm{RMSE}=21.96 \mathrm{mg} / \mathrm{dL}$ \\
\hline C. Araujo-Andrade [32] & $\begin{array}{l}\text { Diffuse } \\
\text { reflectance }\end{array}$ & $900-1700$ & High & $\begin{array}{l}\mathrm{RMSE}=16 \mathrm{mg} / \mathrm{dL} \\
R^{2}=0.74\end{array}$ \\
\hline
\end{tabular}

\section{CONCLUSION}

In this paper, a non-invasive system is developed using NIR LED sensor which helps for regular blood glucose monitoring in an economical way. Experiments are performed using our prototype and commercially glucometer, PPG signals are obtained based on a prototype system and linear regression method used to model the relationship between PPG signals and BGC. The results show that the NIR system is an encouraging method for non-invasive estimation of BGC. In the near future, we will plan an experimental procedure for more subjects. In addition, we will use different wavelength and multiple regression analyses for further improvement of the proposed system. 


\section{REFERENCES}

[1] D. Garcia-Compean, J. O. Jacquez-Quintana, J. A. Gonzalez-Gonzalez, and H. Maldonado-Garza, "Liver cirrhosis and diabetes: Risk factors, pathophysiology, clinical implications and management," World J. Gastroenterol., vol. 15 , no. 3, pp. 280-288, 2009.

[2] M. Mitrović, D. S. Popović, D. T. Naglić, J. N. Paro, T. Ilić, and B. K. Zavišić, "Markers of inflammation and microvascular complications in type 1 diabetes," Cent. Eur. J. Med., vol. 9, no. 6, pp. 748-753, 2014.

[3] International Diabetes Federation, "IDF Diabetes Atlas 6th edn. 2014 update," IDF Diabetes Atlas Sixth edition, 2014.

[4] D. M. Nathan, "Long-term complications of diabetes mellitus," N. Engl. J. Med., vol. 328, no. 23, pp. 1676-1685, 1993.

[5] K. Yamaoka, J. Eguchi, and S. Uno, "Potentiometric Glucose Detection by Paper-based Electrochemical Sensor on CMOS Chip,” Telkomnika, vol. 15, no. 2, p. 836, 2017.

[6] T. Fujimoto et al., "Portable Electrochemical Sensing System Attached to Smartphones and Its Incorporation with Paper-based Electrochemical Glucose Sensor," Int. J. Electr. Comput. Eng., vol. 7, no. 3, 2017.

[7] A. Nawaz, S. Sælid, P. Øhlckers, M. Jacobsen, and M. Nadeem Akram, "Review: Non-Invasive Continuous Blood Glucose Measurement Techniques," J. Bioinforma. Diabetes, pp. 1-27, 2016.

[8] N. Jahangiri, A. Bahrampour, and M. Taraz, "Non-invasive Optical Techniques for determination of blood Glucose levels : A Review Article," vol. 11, no. 2, pp. 224-232, 2014.

[9] K. Prawiroredjo and E. S. Julian, "Comparative study of $940 \mathrm{~nm}$ and $1450 \mathrm{~nm}$ near infrared sensor for glucose concentration monitoring," TELKOMNIKA, vol. 17, no. 2, pp. 981-985, 2019.

[10] J. Yadav, A. Rani, V. Singh, and B. M. Murari, "Design of Low Cost Blood Glucose Sensing System Using Diffused Reflectance Near-Infrared Light," in Networking Communication and Data Knowledge Engineering, Springer, pp. 197-216, 2018.

[11] S. Ramasahayam, K. S. Haindavi, B. Kavala, and S. R. Chowdhury, "Non invasive estimation of blood glucose using near infra red spectroscopy and double regression analysis, " in Proceedings of the International Conference on Sensing Technology, ICST, pp. 627-631, 2013.

[12] S. Ramasahayam, S. H. Koppuravuri, L. Arora, and S. R. Chowdhury, "Noninvasive Blood Glucose Sensing Using Near Infra-Red Spectroscopy and Artificial Neural Networks Based on Inverse Delayed Function Model of Neuron," J. Med. Syst., vol. 39, no. 1, pp. 1-15, 2015.

[13] M. Ionescu, "Measuring and detecting blood glucose by methods non-invasive," in 10th Edition Electronics, Computers and Artificial Intelligence, no. July 2018.

[14] K. Sarkar, D. Ahmad, S. K. Singha, and M. Ahmad, "Design and Implementation of a Noninvasive Blood Glucose Monitoring Device," in 2018 21st International Conference of Computer and Information Technology (ICCIT), pp. 1-5, 2018,

[15] J. S. Maier, S. A. Walker, S. Fantini, M. A. Franceschini, and E. Gratton, "Possible correlation between blood glucose concentration and the reduced scattering coefficient of tissues in the near infrared," Opt. Lett., vol. 19, no. 24, p. 2062, 1994.

[16] P. Narkhede, S. Dhalwar, and B. Karthikeyan, "NIR based non-invasive blood glucose measurement," Indian J. Sci. Technol., vol. 9, no. 41, 2016.

[17] B. Paul, M. P. Manuel, and Z. C. Alex, "Design and development of non invasive glucose measurement system," in Proceedings - ISPTS-1, 1st International Symposium on Physics and Technology of Sensors, pp. 43-46, 2012.

[18] M. N. Ali, M. Benabdellah, and S. Abdelouahed, "Conception and Realization of a Biomedical Acquisition System Dedicated to Measuring the Oxygen Saturation Pulse by Photo Plethysmography Red and Infrared : Application to theCardio- Respiratory Functional Exploration," vol. 4, no. 7, pp. 28-36, 2014.

[19] N. F. A. Jamal, K. A. Sidek, and A. Z. Jusoh, "Portable health monitoring kit using photolethysmogram signal," Indones. J. Electr. Eng. Comput. Sci., vol. 15, no. 2, pp. 638-649, 2019.

[20] N. S. Ali, Z. A. A. Alyasseri, and A. Abdulmohson, "Real-Time Heart Pulse Monitoring Technique Using Wireless Sensor Network and Mobile Application," Int. J. Electr. Comput. Eng., vol. 8, no. 6, p. 5118, 2018.

[21] N. A. Nayan, R. Jaafar, and N. S. Risman, "Development of respiratory rate estimation technique using electrocardiogram and photoplethysmogram for continuous health monitoring," Bull. Electr. Eng. Informatics, vol. 7, no. 3, pp. 487-494, 2018.

[22] H. Lee, J. Lee, W. Jung, and G. Lee, "The periodic moving average filter for removing motion artifacts from PPG signals," Int. J. Control Autom. Syst., vol. 5, no. 6, pp. 701-706, 2007.

[23] V. S. Murthy, S. Ramamoorthy, N. Srinivasan, S. Rajagopal, and M. M. Rao, "Analysis of photoplethysmographic signals of cardiovascular patients," in 2001 Conference Proceedings of the 23rd Annual International Conference of the IEEE Engineering in Medicine and Biology Society, vol. 3, pp. 2204-2207, 2001.

[24] B. Gayathri, K. Sruthi, and K. A. U. Menon, "Non-invasive blood glucose monitoring using near infrared spectroscopy," in Proceedings of the 2017 IEEE International Conference on Communication and Signal Processing, ICCSP 2017, vol. 2018-Janua, pp. 1139-1142, 2017.

[25] W. L. Clarke, D. Cox, L. A. Gonder-Frederick, W. Carter, and S. L. Pohl, "Evaluating clinical accuracy of systems for self-monitoring of blood glucose," Diabetes Care, vol. 10, no. 5, pp. 622-628, 1987.

[26] B. Javid, F. Fotouhi-Ghazvini, and F. Zakeri, "Noninvasive optical diagnostic techniques for mobile blood glucose and bilirubin monitoring," J. Med. Signals Sensors, vol. 8, no. 3, p. 125, 2018.

[27] M. ud din Q, "GSM based Needleless Blood Glucose Monitoring System,” J. Bioeng. Biomed. Sci., vol. 07, no. 03, pp. 7-10, 2017. 
[28] H. Ali, F. Bensaali, and F. Jaber, "Novel Approach to Non-Invasive Blood Glucose Monitoring Based on Transmittance and Refraction of Visible Laser Light," IEEE Access, vol. 5, pp. 9163-9174, 2017.

[29] S. Haxha and J. Jhoja, "Optical Based Noninvasive Glucose Monitoring Sensor Prototype," IEEE Photonics J., vol. 8, no. 6, pp. 1-10, 2016.

[30] X. Li and C. Li, "Research on non-invasive glucose concentration measurement by NIR transmission," in 2015 IEEE International Conference on Computer and Communications (ICCC), pp. 223-228, 2015.

[31] E. Guevara and F. J. González, "Joint optical-electrical technique for noninvasive glucose monitoring," Rev. Mex. Fis., vol. 56, no. 5, pp. 430-434, 2010.

[32] C. Araujo- Andrade, F. Ruiz, J. R. Martínez- Mendoza, and H. Terrones, "Non- invasive in- vivo blood glucose levels prediction using near infrared spectroscopy," in AIP Conference Proceedings, pp. 234-239, 2004.

[33] S. J. Yeh, C. F. Hanna, and O. S. Khalil, "Monitoring blood glucose changes in cutaneous tissue by temperaturemodulated localized reflectance measurements," Clin. Chem., vol. 49, no. 6, pp. 924-934, 2003. 